\title{
Can discovery of hidden charm strange pentaquark states help determine the spins of $P_{c}(4440)$ and $P_{c}(4457)$ ?
}

\author{
Ming-Zhu Liu $\odot,{ }^{1}$ Ya-Wen Pan, ${ }^{2}$ and Li-Sheng Geng ${ }^{2,3,4,5, *}$ \\ ${ }^{1}$ School of Space and Environment, Beihang University, Beijing 100191, China \\ ${ }^{2}$ School of Physics, Beihang University, Beijing 100191, China \\ ${ }^{3}$ Beijing Key Laboratory of Advanced Nuclear Materials and Physics, Beihang University, \\ Beijing 100191, China \\ ${ }^{4}$ School of Physics and Microelectronics, Zhengzhou University, Zhengzhou, Henan 450001, China \\ ${ }^{5}$ Beijing Advanced Innovation Center for Big Data-Based Precision Medicine, \\ School of Medicine and Engineering, Beihang University, Beijing 100191, China
}

(Received 29 November 2020; accepted 13 January 2021; published 5 February 2021)

The pentaquark states $P_{c}(4312), P_{c}(4440)$, and $P_{c}(4457)$ could be nicely arranged into a multiplet of seven molecules of $\bar{D}^{(*)} \Sigma_{c}^{(*)}$ dictated by heavy quark spin symmetry. However, the spins of $P_{c}(4440)$ and $P_{c}(4457)$ are not yet fully determined. We employ the contact-range effective field theory to investigate the SU(3)-flavor counterparts of $\bar{D}^{(*)} \Sigma_{c}^{(*)}$ and study the possibility of whether their discovery could help determine the spins of $P_{c}(4457)$ and $P_{c}(4440)$. We find the existence of a complete hidden charm strange multiplet of $\bar{D}^{(*)} \Xi_{c}^{(\prime) *}$ molecules, irrespective of the spins of $P_{c}(4440)$ and $P_{c}(4457)$. On the other hand, we find that although molecules of $\bar{D}^{(*)} \Xi_{c}$ are also likely, depending on the realization of the underlying dynamics, their discovery could be more useful for determining the spins of $P_{c}(4440)$ and $P_{c}(4457)$ and for telling us how heavy quark and light quark interactions depend on the spin of the light quark pair.

DOI: 10.1103/PhysRevD.103.034003

\section{INTRODUCTION}

In 2015, the LHCb Collaboration reported two reasonance states, $P_{c}(4380)$ and $P_{c}(4450)$, in the $J / \psi p$ invariant mass spectrum of the $\Lambda_{b} \rightarrow J / \psi p K$ decay [1], whose mass and decay width are

$$
\begin{aligned}
M_{P_{4380}} & =4380 \pm 8 \pm 29 \mathrm{MeV} \\
\Gamma_{P_{4380}} & =205 \pm 18 \pm 86 \mathrm{MeV}, \\
M_{P_{4450}} & =4449.8 \pm 1.7 \pm 2.5 \mathrm{MeV} \\
\Gamma_{P_{4450}} & =39 \pm 5 \pm 19 \mathrm{MeV},
\end{aligned}
$$

respectively. Because of the closeness of $P_{c}(4450)$ to the mass threshold of $\bar{D}^{*} \Sigma_{c}$, as well as its narrow decay width, it is often suggested that it is a hadronic molecule [2-7]. It should be noted that the existence of $\bar{D}^{(*)} \Sigma_{c}^{(*)}$ molecules had been predicted before the LHCb discovery [8-13].

\footnotetext{
* Corresponding author. lisheng.geng@buaa.edu.cn

Published by the American Physical Society under the terms of the Creative Commons Attribution 4.0 International license. Further distribution of this work must maintain attribution to the author(s) and the published article's title, journal citation, and DOI. Funded by SCOAP .
}

In 2019 the LHCb Collaboration updated their analysis with a dataset almost 10 times larger and found that the previous $P_{c}(4450)$ state splits into two states, $P_{c}(4440)$ and $P_{c}(4457)$, and in addition that a new narrow state $P_{c}(4312)$ [14] emerges just below the $\bar{D} \Sigma_{c}$ threshold. Their masses and decay widths are

$$
\begin{aligned}
M_{P_{4312}} & =4311.9 \pm 0.7_{-0.6}^{+6.8} \mathrm{MeV} \\
\Gamma_{P_{4312}} & =9.8 \pm 2.7_{-4.5}^{+3.7} \mathrm{MeV} \\
M_{P_{4440}} & =4440.3 \pm 1.3_{-4.7}^{+4.1} \mathrm{MeV} \\
\Gamma_{P_{4440}} & =20.6 \pm 4.9_{-10.1}^{+8.7} \mathrm{MeV} \\
M_{P_{4457}} & =4457.3 \pm 0.6_{-1.7}^{+4.1} \mathrm{MeV} \\
\Gamma_{P_{4457}} & =6.4 \pm 2.0_{-1.9}^{+5.7} \mathrm{MeV} .
\end{aligned}
$$

In our previous work we showed that these states can be understood as hadronic molecules in both an effective field theory (EFT) approach and the one boson exchange (OBE) model $[15,16]$. Although at present the molecular interpretation might be the favored one [17-24], there are other explanations, e.g., hadrocharmonium [25], compact pentaquark states [26-32], or virtual states [33]. See Refs. [34-36] for the latest reviews. As argued in Ref. [37], the most crucial (but still missing) information to disentangle different interpretations is their spins. 
Symmetry is a core concept in particle physics and plays an important role in studying heavy hadronic molecules. Two symmetries relevant to this work are heavy quark spin symmetry (HQSS) and heavy antiquark diquark symmetry (HADS). The HQSS dictates that the strong interaction is independent of the spin of the heavy quark in the limit of heavy quark masses [38,39], which provides a natural explanation to the mass difference between $\left(D, D^{*}\right)$ and $\left(B, B^{*}\right)$, as well as that of their baryon counterparts. Applying HQSS to studying heavy hadronic molecules, $D_{s 0}(2317)$ and $D_{s 1}(2460)$ can be naturally interpreted as $D K$ and $D^{*} K$ molecules of spin doublets [40,41]. The HQSS also plays an important role in describing the three pentaquark states $P_{c}(4312), P_{c}(4440)$, and $P_{c}(4457)$ as $\bar{D}^{*} \Sigma_{c}$ molecules. In particular, we obtained a complete multiplet of hadronic molecules in the $\bar{D}^{(*)} \Sigma_{c}^{(*)}$ system in both the EFT approach and the OBE model $[15,16]$, which was later corroborated by many studies [17,20-23]. In addition to HQSS, HADS has also been used to study heavy hadronic molecules [37,42] and to estimate the coupling of a doubly charmed baryon to a pion [43]. In Refs. [37,42], we extended the $\bar{D}^{(*)} \Sigma_{c}^{(*)}$ system to the $\Sigma_{c}^{(*)} \Xi_{c c}^{(*)}$ system via HADS and predicted a complete multiplet of triply charmed hadronic molecules. In particular, we pointed out that the splittings of $\Xi_{c c} \Sigma_{c}$ states are correlated with the spins of $P_{c}(4440)$ and $P_{c}(4457)$, which, given the fact that the former can be much more easily simulated on the lattice [44], provides the possibility of determining the spins of the latter in a model independent way.

Along these lines, in this work, we explore whether one can relate the pentaquark states $P_{c}(4440)$ and $P_{c}(4457)$ to other states via symmetries such that their discovery could shed light on the nature of the $P_{c}$ states, particularly their spin in the molecular picture. The symmetry of current interest is SU(3)flavor symmetry, which relates the hidden charm states to hidden charm strange states. As a result, the discovery of the latter will shed light on the nature of the former.

Before $P_{c}(4380)$ and $P_{c}(4450)$ were discovered by the LHCb Collaboration, Wu et al. had already predicted four hidden charm strange pentaquark states through a local hidden-gauge Lagrangian in combination with unitary techniques in coupled channels [8]. After the discovery of three pentaquark states in 2019, the study was updated and ten hidden charm strange pentaquark states were predicted [45]. In Ref. [46] two partners of $P_{c}(4380)$ and $P_{c}(4450)$ were predicted in the OBE model. More recently, Wang et al. also predicted the existence of ten hidden charm strange pentaquark states in the chiral effective field theory [47]. The discovery potential of hidden charm strange pentaquark states in the $J / \psi \Lambda$ invariant mass spectra of the $\Xi_{b} \rightarrow$ $J / \psi \Lambda K$ and $\Lambda_{b} \rightarrow J / \psi \Lambda K$ decays have been explored $[48,49]$, as have their partial decay widths [50].

In this work we adopt an EFT approach to study possible hidden charm strange molecules of $\bar{D}^{(*)} \Xi_{c}$ and $\bar{D}^{(*)} \Xi_{c}^{(\prime) *}$, which can be regarded as the $\mathrm{SU}(3)$-flavor counterparts of
$\bar{D}^{(*)} \Sigma_{c}^{(*)}$. In particular, we focus on the correlation between hidden charm and hidden charm strange molecules, which can not only help check the molecular interpretation of $P_{c}(4440)$ and $P_{c}$ (4457) but also help determine their spin ordering if the hidden charm strange pentaquark states are discovered by either experiments or lattice QCD calculations, which is analogous to the correlation dictated by HADS as shown in Refs. [37,42].

The manuscript is structured as follows. In Sec. II we present the details of the contact-range potential of $\bar{D}^{(*)} \Xi_{c}$ and $\bar{D}^{(*)} \Xi_{c}^{(\prime) *}$ according to heavy quark spin symmetry and $\mathrm{SU}(3)$-flavor symmetry. In Sec. III we give the full spectrum of hidden charm strange molecules. Finally we present our conclusions in Sec. IV.

\section{THEORETICAL FORMALISM}

Here we explain how to determine the $\bar{D}^{(*)} \Xi_{c}$ and $\bar{D}^{(*)} \Xi_{c}^{(\prime) *}$ interactions and study the likely existence of hidden charm strange pentaquark states. Following Refs. [15,37], we determine the $\bar{D}^{(*)} \Xi_{c}$ and $\bar{D}^{(*)} \Xi_{c}^{(\prime) *}$ interactions in an EFT approach. One should note that we consider only the leading order contact-range potentials because our previous studies indicated that pion exchange contributions are perturbative in the charm sector [51,52].

Because the $\Xi_{c}^{\prime(*)}$ and $\Sigma_{c}^{(*)}$ baryons belong to the same $\mathrm{SU}(3)$ group representation, the interactions of $\bar{D}^{(*)} \Xi_{c}^{(\prime) *}$ and $\bar{D}^{(*)} \Sigma_{c}^{(*)}$ are the same in the heavy quark mass and SU (3)-flavor symmetry limits. As a result, the same two low energy constants are needed to account for the contact-range potentials of $\bar{D}^{(*)} \Sigma_{c}^{(*)}$ and $\bar{D}^{(*)} \Xi_{c}^{\prime(*)}, C_{a}$ and $C_{b}$, namely,

$$
V=C_{a}+\sigma_{1} \cdot \sigma_{2} C_{b} .
$$

There are seven combinations for the $\bar{D}^{(*)} \Xi_{c}^{\prime(*)}$ system according to HQSS, whose potential can be written as

$$
\begin{gathered}
V\left(\bar{D} \Xi_{c}^{\prime}, J=\frac{1}{2}\right)=C_{a}, \\
V\left(\bar{D} \Xi_{c}^{*}, J=\frac{3}{2}\right)=C_{a}, \\
V\left(\bar{D}^{*} \Xi_{c}^{\prime}, J=\frac{1}{2}\right)=C_{a}-\frac{4}{3} C_{b}, \\
V\left(\bar{D}^{*} \Xi_{c}^{\prime}, J=\frac{3}{2}\right)=C_{a}+\frac{2}{3} C_{b}, \\
V\left(\bar{D}^{*} \Xi_{c}^{*}, J=\frac{1}{2}\right)=C_{a}-\frac{5}{3} C_{b},
\end{gathered}
$$




$$
\begin{aligned}
& V\left(\bar{D}^{*} \Xi_{c}^{*}, J=\frac{3}{2}\right)=C_{a}-\frac{2}{3} C_{b}, \\
& V\left(\bar{D}^{*} \Xi_{c}^{*}, J=\frac{5}{2}\right)=C_{a}+C_{b} .
\end{aligned}
$$

The interaction of the $\bar{D}^{(*)} \Xi_{c}$ system is different from that of $\bar{D}^{(*)} \Xi_{c}^{(\prime) *}$ because the spin of the light quark pair in $\Xi_{c}$ is 0 and that in $\Xi_{c}^{\prime(*)}$ is 1 . In terms of HQSS, the contactrange potential between $\bar{D}^{(*)}$ and $\Xi_{c}^{\prime *}$ can be denoted as $F_{1 / 2}$ and $F_{3 / 2}$ via the coupling of the light quark spins, i.e., $1 / 2 \otimes 1=1 / 2 \oplus 3 / 2$. Applying the same approach to $\bar{D}^{(*)} \Xi_{c}$, the corresponding potential can be represented by one low energy constant $F_{1 / 2}^{\prime}$, which is from the light quark spin coupling, $1 / 2 \otimes 0=1 / 2$.

In principle, $F_{1 / 2}$ and $F_{1 / 2}^{\prime}$ can be different, and there is no reliable way to relate them. In this work, we will take two assumptions and rely on future experiments or lattice QCD simulations to verify which assumption is realized in nature.

First, we assume that $F_{1 / 2}$ in the $\bar{D}^{(*)} \Xi_{c}^{(\prime) *}$ system and $F_{1 / 2}^{\prime}$ in the $\bar{D}^{*} \Xi_{c}$ system are the same, which is denoted as case $\mathrm{I}$ in the following. To find the relationship between the $\bar{D}^{(*)} \Xi_{c}^{(\prime) *}$ system and the $\bar{D}^{(*)} \Xi_{c}$ system, the couplings of $F_{1 / 2}$ and $F_{3 / 2}$ can be rewritten as $F_{1 / 2}=C_{a}-2 C_{b}$ and $F_{3 / 2}=C_{a}+C_{b}$. Then the contact-range potentials of $\bar{D}^{(*)} \Xi_{c}$ have the following form:

$$
\begin{aligned}
& V\left(\bar{D}^{\Xi_{c}}, J=\frac{1}{2}\right)=C_{a}-2 C_{b}, \\
& V\left(\bar{D}^{*} \Xi_{c}, J=\frac{1}{2}\right)=C_{a}-2 C_{b}, \\
& V\left(\bar{D}^{*} \Xi_{c}, J=\frac{3}{2}\right)=C_{a}-2 C_{b} .
\end{aligned}
$$

One should note that the $\bar{D}^{(*)} \Xi_{c}^{\prime(*)}$ contact-range potential can also be denoted as $F_{1 / 2}$ and $F_{3 / 2}$ in terms of HQSS.

Second, we assume that $F_{1 / 2}^{\prime}$ is not the same as $F_{1 / 2}$ and turn to some phenomenological methods for help, which are denoted as case II. One such phenomenological method is the local hidden-gauge approach. According to Ref. [45], the contact-range potential of the $\bar{D}^{(*)} \Xi_{c}$ system is written as

$$
\begin{gathered}
V\left(\bar{D} \Xi_{c}, J=\frac{1}{2}\right)=C_{a}, \\
V\left(\bar{D}^{*} \Xi_{c}, J=\frac{1}{2}\right)=C_{a},
\end{gathered}
$$

$$
V\left(\bar{D}^{*} \Xi_{c}, J=\frac{3}{2}\right)=C_{a} .
$$

Clearly, the strength is the same for all the three channels, but it is different from that of case I. We hope that future experimental or lattice QCD data will tell which case is realized in nature.

To search for bound states, we solve the LippmannSchwinger equation with contact-range potentials

$$
\phi(k)+\int \frac{d^{3} p}{(2 \pi)^{3}}\langle k|V| p\rangle \frac{\phi(p)}{B+\frac{p^{2}}{2 \mu}}=0,
$$

where $\phi(k)$ is the vertex function, $B$ the binding energy, and $\mu$ the reduced mass. To solve the equation we have to regularize the contact potential

$$
\left\langle p\left|V_{\Lambda}\right| p^{\prime}\right\rangle=C(\Lambda) f\left(\frac{p}{\Lambda}\right) f\left(\frac{p^{\prime}}{\Lambda}\right),
$$

with $\Lambda$ the cutoff, $f(x)$ a regular function, and $C(\Lambda)$ the running coupling constant. A typical choice of the cutoff is $\Lambda=0.5-1 \mathrm{GeV}$, while for the regulator we choose a Gaussian type $f(x)=e^{-x^{2}}$. In this work, we consider only $S$-wave contact contribution; thus the integral equation simplifies to

$$
1+C(\Lambda) \frac{\mu}{\pi^{2}} \int_{0}^{\infty} d q e^{-2 \frac{q^{2}}{\Lambda^{2}}} \frac{q^{2}}{B+\frac{\vec{q}^{2}}{2 \mu}}=0 .
$$

\section{NUMERICAL RESULTS AND DISCUSSIONS}

From HQSS and SU(3)-flavor symmetry, we express the contact potentials of $\bar{D}^{(*)}$ and $\Xi_{c}^{\prime(*)}$ by two coupling constants, $C_{a}$ and $C_{b}$, which are the only two unknown inputs for us to obtain the binding energies of mesonbaryon systems under consideration. In our previous works, we proposed that $P_{c}(4440)$ and $P_{c}(4457)$ are bound states of $\bar{D}^{*} \Sigma_{c}$ with either spin $1 / 2$ or spin $3 / 2$ and negative party, and the two LECs $C_{a}$ and $C_{b}$ have been determined by fitting to the masses of $P_{c}(4440)$ and $P_{c}(4457)$. As a result, in the following, we study two scenarios, Scenario A, where $P_{c}(4440)$ and $P_{c}(4457)$ have $1 / 2^{-}$and $3 / 2^{-}$, and scenario $B$, where they have the opposite. With $\Lambda=1 \mathrm{GeV}$ we obtain $C_{a}=-20.14 \mathrm{GeV}^{-2}$ and $C_{b}=2.73 \mathrm{GeV}^{-2}$ for scenario A and $C_{a}=-21.96 \mathrm{GeV}^{-2}$ and $C_{b}=-2.73 \mathrm{GeV}^{-2}$ for scenario $\mathrm{B}$, and with $\Lambda=$ $0.5 \mathrm{GeV} C_{a}=-55.54 \mathrm{GeV}^{-2}$ and $C_{b}=-13.90 \mathrm{GeV}^{-2}$ for scenario $\mathrm{A}$ and $C_{a}=-64.80 \mathrm{GeV}^{-2}$ and $C_{b}=$ $-13.90 \mathrm{GeV}^{-2}$ for scenario $\mathrm{B}$. The results are displayed in Table I. We find that all seven states in the $\bar{D}^{(*)} \Xi_{c}^{(\prime) *}$ system bind in both scenarios $\mathrm{A}$ and $\mathrm{B}$, which is consistent with Refs. [45,47]. This indicates that these kinds of hidden 
TABLE I. Bound states of a singly charmed baryon and a singly charmed antimeson, obtained in the contact-range effective field theory with the two constants fixed by reproducing $P_{c}(4440)$ and $P_{c}(4457)$ as $1 / 2^{-}$and $3 / 2^{-}$molecules (scenario A) and $3 / 2^{-}$and $1 / 2^{-}$(scenario B), respectively, with cutoffs of 0.5 and $1 \mathrm{GeV}$, and the $25 \%$ uncertainty caused by SU(3)-flavor symmetry and heavy quark spin symmetry breaking for the contact-range potentials.

\begin{tabular}{ccccccccc}
\hline \hline State & $J^{P}$ & $\Lambda(\mathrm{GeV})$ & B. E(A) & Mass(A) & B. E(B) & Mass(B) & Ref. [45] & Ref. [47] \\
\hline $\bar{D} \Xi_{c}^{\prime}$ & $1 / 2^{-}$ & $1(0.5)$ & $8.5_{-8.4}^{+17.4}\left(9.3_{-6.7}^{+8.7}\right)$ & $4437(4436)$ & $14.0_{-12.8}^{+21.7}\left(14.9_{-9.3}^{+11.4}\right)$ & $4431(4430)$ & 4436.7 & 4423.7 \\
$\bar{D} \Xi_{c}^{*}$ & $3 / 2^{-}$ & $1(0.5)$ & $9.0_{-8.8}^{+17.7}\left(9.5_{-6.7}^{+7.8}\right)$ & $4504(4504)$ & $14.7_{-13.3}^{+12.9}\left(15.2_{-9.4}^{+11.4}\right)$ & $4499(4498)$ & 4506.99 & 4502.9 \\
$\bar{D}^{*} \Xi_{c}^{\prime}$ & $1 / 2^{-}$ & $1(0.5)$ & $23.4_{-18.9}^{+27.0}\left(22.5_{-12.3}^{+14.2}\right)$ & $4563(4564)$ & $5.6_{\dot{+}}^{+14.3}\left(5.2_{-4.9}^{+6.4}\right)$ & $4581(4581)$ & 4580.96 & 4568.7 \\
$\bar{D}^{*} \Xi_{c}^{\prime}$ & $3 / 2^{-}$ & $1(0.5)$ & $5.6_{\dagger}^{+14.3}\left(5.2_{-4.3}^{+6.4}\right)$ & $4581(4581)$ & $23.4_{-18.8}^{+27.0}\left(22.5_{-12.3}^{+14.2}\right)$ & $4563(4564)$ & 4580.96 & 4582.3 \\
$\bar{D}^{*} \Xi_{c}^{*}$ & $1 / 2^{-}$ & $1(0.5)$ & $28.0_{-21.4}^{+29.4}\left(26.3_{-13.7}^{+15.5}\right)$ & $4627(4628)$ & $4.0_{\dagger}^{+12.5}\left(3.3_{-3.0}^{+5.1}\right)$ & $4651(4651)$ & 4650.86 & 4635.4 \\
$\bar{D}^{*} \Xi_{c}^{*}$ & $3 / 2^{-}$ & $1(0.5)$ & $17.2_{-14.9}^{+23.2}\left(16.4_{-9.8}^{+11.6}\right)$ & $4637(4638)$ & $11.1_{-10.5}^{+18.9}\left(10.5_{-7.2}^{+9.1}\right)$ & $4643(4644)$ & 4650.58 & 4644.4 \\
$\bar{D}^{*} \Xi_{c}^{*}$ & $5 / 2^{-}$ & $1(0.5)$ & $4.0_{\dagger}^{+12.5}\left(3.3_{-3.0}^{+5.1}\right)$ & $4651(4651)$ & $28.0_{-21.4}^{+29.4}\left(26.3_{-13.7}^{+15.5}\right)$ & $4627(4628)$ & 4650.56 & 4651.7 \\
\hline \hline
\end{tabular}

charm strange molecules must exist. In addition their results favor scenario A, namely, $P_{c}(4440)$ and $P_{c}(4457)$ have spins $1 / 2$ and $3 / 2$, respectively.

From Table I one can easily see that our results are almost independent of the cutoff. Compared to the results of the $\Xi_{c c}^{(*)} \Sigma_{c}^{(*)}$ bound states [37], the cutoff dependence is much weaker, which implies that SU(3)-flavor symmetry is less broken than HADS. To estimate the breaking of SU(3)flavor symmetry, we adopted $20 \%$ uncertainty, estimated from the ratio of $f_{D}$ and $f_{D_{s}}$ calculated by lattice QCD [53-56]. Also taking into account heavy quark spin symmetry breaking at the order of $\delta_{\mathrm{HQSS}}=15 \%$, we assign a total uncertainty of $\delta=\sqrt{\delta_{\mathrm{HQSS}}^{2}+\delta_{S U(3)}^{2}}=25 \%$ for the $\bar{D}^{(*)} \Xi_{c}^{\prime(*)}$ interaction. After taking into account the large uncertainties caused by symmetry breaking, all the states still bind with a cutoff of $0.5 \mathrm{GeV}$ and two states can become unbound with a cutoff of $1 \mathrm{GeV}$, while the spin ordering remains unchanged, which suggests that the hidden charm strange molecules must exist if $P_{c}(4312)$, $P_{c}(4440)$, and $P_{c}(4457)$ are (dominantly) $\bar{D}^{(*)} \Sigma_{c}$ molecules. Compared with their hidden charm partners, these states could be detected in the $J / \psi \Lambda$ invariant mass spectra of the $\Xi_{b} \rightarrow J / \psi \Lambda K$ decay. If these states are discovered experimentally, it not only will further enrich the family of hadronic molecules but also help to determine the spin ordering of $P_{c}(4440)$ and $P_{c}$ (4457).

For the $\bar{D}^{(*)} \Xi_{c}$ system, case I assumes that the coupling $F_{1 / 2}$ is the same as the coupling $F_{1 / 2}^{\prime}$, and therefore the contact-range potential of $\bar{D}^{(*)} \Xi_{c}$ can also be written as combinations of $C_{a}$ and $C_{b}$. Thus we can calculate the binding energies of the $\bar{D}^{(*)} \Xi_{c}$ systems in the two scenarios $\mathrm{A}$ and $\mathrm{B}$ as well. The results are shown in Table II. Interestingly, we note that the binding energies in scenario A are much larger than their counterparts in scenario B. To estimate the uncertainty of SU(3)-flavor symmetry and the assumed equality of $F_{1 / 2}$ and $F_{1 / 2}^{\prime}$, we consider a larger uncertainty of $30 \%$ into the $C_{a}$ and $C_{b}$ values [57]. We find that the $\bar{D}^{(*)} \Xi_{c}$ systems in scenario B can become unbound while they still bind in scenario A, which indicates that the $\bar{D}^{(*)} \Xi_{c}$ hidden charm strange molecules exist in scenario A and may not necessarily exist in scenario B. If such molecules are found experimentally, it implies that the spins of $P_{c}(4440)$ and $P_{c}(4457)$ are more likely to be $1 / 2$ and $3 / 2$, respectively.

TABLE II. (Likely) bound states of a singly charmed baryon and a singly charmed antimeson, obtained in the contact-range effective field theory, with the two constants fixed by reproducing $P_{c}(4440)$ and $P_{c}(4457)$ as either $1 / 2^{-}$and $3 / 2^{-}$molecules (scenario A) or $3 / 2^{-}$and $1 / 2^{-}$molecules (scenario B), respectively. with cutoffs of 0.5 and $1 \mathrm{GeV}$, and the $30 \%$ uncertainty estimated using the light meson saturation approach for the contact-range potentials. Cases I and II differ from each other in how $F_{1 / 2}^{\prime}$ is determined.

\begin{tabular}{cccccccccc}
\hline \hline & State & $J^{P}$ & $\Lambda(\mathrm{GeV})$ & B. E(A) & Mass(A) & B. E(B) & Mass(B) & {$[45]$} & {$[47]$} \\
\hline I & $\bar{D} \Xi_{c}$ & $1 / 2^{-}$ & $1(0.5)$ & $26.3_{-24.3}^{+36.1}\left(27.4_{-16.9}^{+19.6}\right)$ & $4310(4309)$ & $0.9_{\dagger}^{+10.5}\left(1.0_{\dagger}^{+4.1}\right)$ & $4335(4335)$ & 4276.59 & 4319.4 \\
& $\bar{D}^{*} \Xi_{c}$ & $1 / 2^{-}$ & $1(0.5)$ & $29.5_{-25.4}^{+37.4}\left(28.8_{-17.4}^{+20.0}\right)$ & $4448(4449)$ & $1.6_{\dagger}^{+12.0}\left(1.3_{\dagger}^{+4.5}\right)$ & $4476(4476)$ & 4429.84 & 4456.9 \\
& $\bar{D}^{*} \Xi_{c}$ & $3 / 2^{-}$ & $1(0.5)$ & $29.5_{-25.4}^{+37.4}\left(28.8_{-17.4}^{+20.0}\right)$ & $4448(4449)$ & $1.6_{\dagger}^{+12.0}\left(1.3_{\dagger}^{+4.5}\right)$ & $4476(4476)$ & 4429.84 & 4463.0 \\
II & $\bar{D} \Xi_{c}$ & $1 / 2^{-}$ & $1(0.5)$ & $7.7_{\dagger}^{+20.9}\left(8.9_{-7.4}^{+10.5}\right)$ & $4329(4327)$ & $13.0_{-12.9}^{+26.0}\left(14.4_{-10.6}^{+13.6}\right)$ & $4335(4321)$ & 4276.59 & 4319.4 \\
& $\bar{D}^{*} \Xi_{c}$ & $1 / 2^{-}$ & $1(0.5)$ & $9.6_{\dagger}^{+22.4}\left(9.8_{-7.9}^{+10.8}\right)$ & $4468(4468)$ & $15.4_{-15.0}^{+28.4}\left(15.5_{-11.0}^{+14.0}\right)$ & $4462(4462)$ & 4429.84 & 4456.9 \\
& $\bar{D}^{*} \Xi_{c}$ & $3 / 2^{-}$ & $1(0.5)$ & $9.6_{\dagger}^{+22.4}\left(9.8_{-7.9}^{+10.8}\right)$ & $4468(4468)$ & $15.4_{-15.0}^{+28.4}\left(15.5_{-11.0}^{+14.0}\right)$ & $4462(4462)$ & 4429.84 & 4463.0 \\
\hline \hline
\end{tabular}


In case II we estimate the coupling $F_{1 / 2}^{\prime}$ by turning to the local hidden-gauge approach. As shown above, the value is different from that of case I. The corresponding results are tabulated in Table II. We find that the differences between the binding energies in scenario $\mathrm{A}$ and those in scenario B become less extreme, which implies that we cannot discriminate between the spins of $P_{c}(4440)$ and $P_{c}(4457)$ in case II. As a result, it may not help much to derive the spin ordering of $P_{c}(4457)$ and $P_{c}(4440)$, even if these states are discovered experimentally. On the other hand, their discovery does help to reveal more of the nature of the hidden charm pentaquark states.

\section{SUMMARY}

In this work, assuming that $P_{c}(4312), P_{c}(4440)$, and $P_{c}(4457)$ are $\bar{D}^{(*)} \Sigma_{c}$ molecules, we employed a contactrange effective field theory approach satisfying HQSS and SU(3)-flavor symmetry to study the likely existence of hidden charm strange molecules, with the main purpose being to determine the spin ordering of $P_{c}(4440)$ and $P_{c}(4457)$. To estimate the uncertainty caused by the breaking of these symmetries, we considered a $25 \%$ breaking for $\bar{D}^{(*)} \Xi_{c}^{(\prime) *}$ and a $30 \%$ breaking for $\bar{D}^{(*)} \Xi_{c}$ in our study. Our results showed that there exists a complete multiplet of hadronic molecules in the $\bar{D}^{(*)} \Xi_{c}^{\prime(*)}$ system, irrespective of the spins of $P_{c}(4440)$ and $P_{c}(4457)$.

Assuming that the couplings $F_{1 / 2}$ and $F_{1 / 2}^{\prime}$ are the same, the existence of $\bar{D}^{(*)} \Xi_{c}$ molecules is likely only if $P_{c}(4457)$ has spin $3 / 2$ and $P_{c}(4440)$ has spin $1 / 2$. As a result, the discovery of these states can help one to determine the spins of $P_{c}(4440)$ and $P_{c}(4457)$ in the molecular picture from an EFT perspective. On the other hand, using the hiddengauge approach to infer the coupling of $F_{1 / 2}^{\prime}, \bar{D}^{(*)} \Xi_{c}$ molecules exist irrespective of the spins of $P_{c}(4440)$ and $P_{c}$ (4457), which thus offers little help in determining their spins.

\section{ACKNOWLEDGMENTS}

This work is partly supported by the National Natural Science Foundation of China under Grants No. 11735003, No. 11975041, and No. 11961141004, and by the Fundamental Research Funds for the Central Universities.

Note added.-In a recent talk, the LHCb Collaboration reported the likely existence of a hidden charm strange pentaquark $P_{c s}(4459)$ with a statistical significance of $3.1 \sigma$ [58], which has inspired a number of theoretical studies $[57,59,60]$.
[1] R. Aaij et al. (LHCb Collaboration), Phys. Rev. Lett. 115, 072001 (2015).

[2] R. Chen, X. Liu, X.-Q. Li, and S.-L. Zhu, Phys. Rev. Lett. 115, 132002 (2015).

[3] L. Roca, J. Nieves, and E. Oset, Phys. Rev. D 92, 094003 (2015).

[4] J. He, Phys. Lett. B 753, 547 (2016).

[5] K. Azizi, Y. Sarac, and H. Sundu, Phys. Rev. D 95, 094016 (2017).

[6] L. Geng, J. Lu, and M. P. Valderrama, Phys. Rev. D 97, 094036 (2018).

[7] K. Azizi, Y. Sarac, and H. Sundu, Phys. Lett. B 782, 694 (2018).

[8] J.-J. Wu, R. Molina, E. Oset, and B. S. Zou, Phys. Rev. Lett. 105, 232001 (2010).

[9] J.-J. Wu, R. Molina, E. Oset, and B. S. Zou, Phys. Rev. C 84, 015202 (2011).

[10] C. W. Xiao, J. Nieves, and E. Oset, Phys. Rev. D 88, 056012 (2013).

[11] M. Karliner and J. L. Rosner, Phys. Rev. Lett. 115, 122001 (2015).

[12] W. L. Wang, F. Huang, Z. Y. Zhang, and B. S. Zou, Phys. Rev. C 84, 015203 (2011).

[13] Z.-C. Yang, Z.-F. Sun, J. He, X. Liu, and S.-L. Zhu, Chin. Phys. C 36, 6 (2012).
[14] R. Aaij et al. (LHCb Collaboration), Phys. Rev. Lett. 122, 222001 (2019).

[15] M.-Z. Liu, Y.-W. Pan, F.-Z. Peng, M. Sánchez Sánchez, L.-S. Geng, A. Hosaka, and M. Pavon Valderrama, Phys. Rev. Lett. 122, 242001 (2019).

[16] M.-Z. Liu, T.-W. Wu, M. Pavon Valderrama, J.-J. Xie, and L.-S. Geng, Phys. Rev. D 99, 094018 (2019).

[17] C. W. Xiao, J. Nieves, and E. Oset, Phys. Rev. D 100, 014021 (2019).

[18] C.-J. Xiao, Y. Huang, Y.-B. Dong, L.-S. Geng, and D.-Y. Chen, Phys. Rev. D 100, 014022 (2019).

[19] S. Sakai, H.-J. Jing, and F.-K. Guo, Phys. Rev. D 100, 074007 (2019).

[20] Y. Yamaguchi, H. García-Tecocoatzi, A. Giachino, A. Hosaka, E. Santopinto, S. Takeuchi, and M. Takizawa, Phys. Rev. D 101, 091502 (2020).

[21] M.-Z. Liu, T.-W. Wu, M. Sánchez Sánchez, M. P. Valderrama, L.-S. Geng, and J.-J. Xie, arXiv:1907.06093.

[22] M. Pavon Valderrama, Phys. Rev. D 100, 094028 (2019).

[23] M.-L. Du, V. Baru, F.-K. Guo, C. Hanhart, U.-G. Meißner, J. A. Oller, and Q. Wang, Phys. Rev. Lett. 124, 072001 (2020).

[24] K. Azizi, Y. Sarac, and H. Sundu, arXiv:2011.05828.

[25] M. I. Eides, V. Y. Petrov, and M. V. Polyakov, Mod. Phys. Lett. A 35, 2050151 (2020). 
[26] A. Ali and A. Ya. Parkhomenko, Phys. Lett. B 793, 365 (2019).

[27] H. Mutuk, Chin. Phys. C 43, 093103 (2019).

[28] Z.-G. Wang, Int. J. Mod. Phys. A 35, 2050003 (2020).

[29] J.-B. Cheng and Y.-R. Liu, Phys. Rev. D 100, 054002 (2019).

[30] X.-Z. Weng, X.-L. Chen, W.-Z. Deng, and S.-L. Zhu, Phys. Rev. D 100, 016014 (2019).

[31] R. Zhu, X. Liu, H. Huang, and C.-F. Qiao, Phys. Lett. B 797, 134869 (2019).

[32] A. Pimikov, H.-J. Lee, and P. Zhang, Phys. Rev. D 101, 014002 (2020).

[33] C. Fernández-Ramírez, A. Pilloni, M. Albaladejo, A. Jackura, V. Mathieu, M. Mikhasenko, J. A. Silva-Castro, and A. P. Szczepaniak (JPAC Collaboration), Phys. Rev. Lett. 123, 092001 (2019).

[34] Y.-R. Liu, H.-X. Chen, W. Chen, X. Liu, and S.-L. Zhu, Prog. Part. Nucl. Phys. 107, 237 (2019).

[35] N. Brambilla, S. Eidelman, C. Hanhart, A. Nefediev, C.-P. Shen, C. E. Thomas, A. Vairo, and C.-Z. Yuan, Phys. Rep. 873, 1 (2020).

[36] F.-K. Guo, X.-H. Liu, and S. Sakai, Prog. Part. Nucl. Phys. 112, 103757 (2020).

[37] Y.-W. Pan, M.-Z. Liu, F.-Z. Peng, M. Sánchez Sánchez, L.-S. Geng, and M. Pavon Valderrama, Phys. Rev. D 102, 011504 (2020).

[38] N. Isgur and M. B. Wise, Phys. Lett. B 232, 113 (1989).

[39] N. Isgur and M. B. Wise, Phys. Lett. B 237, 527 (1990).

[40] F.-K. Guo, P.-N. Shen, and H.-C. Chiang, Phys. Lett. B 647, 133 (2007).

[41] M. Altenbuchinger, L. S. Geng, and W. Weise, Phys. Rev. D 89, 014026 (2014).

[42] Y.-W. Pan, M.-Z. Liu, and L.-S. Geng, Phys. Rev. D 102, 054025 (2020).
[43] M.-Z. Liu, Y. Xiao, and L.-S. Geng, Phys. Rev. D 98, 014040 (2018).

[44] P. Junnarkar and N. Mathur, Phys. Rev. Lett. 123, 162003 (2019).

[45] C. Xiao, J. Nieves, and E. Oset, Phys. Lett. B 799, 135051 (2019).

[46] R. Chen, J. He, and X. Liu, Chin. Phys. C 41, 103105 (2017).

[47] B. Wang, L. Meng, and S.-L. Zhu, Phys. Rev. D 101, 034018 (2020).

[48] J.-X. Lu, E. Wang, J.-J. Xie, L.-S. Geng, and E. Oset, Phys. Rev. D 93, 094009 (2016).

[49] H.-X. Chen, L.-S. Geng, W.-H. Liang, E. Oset, E. Wang, and J.-J. Xie, Phys. Rev. C 93, 065203 (2016).

[50] C.-W. Shen, J.-J. Wu, and B.-S. Zou, Phys. Rev. D 100, 056006 (2019).

[51] M. Valderrama, Phys. Rev. D 85, 114037 (2012).

[52] J.-X. Lu, L.-S. Geng, and M. P. Valderrama, Phys. Rev. D 99, 074026 (2019).

[53] E. Follana, C. Davies, G. Lepage, and J. Shigemitsu (HPQCD and UKQCD Collaborations), Phys. Rev. Lett. 100, 062002 (2008).

[54] P. A. Boyle, L. Del Debbio, A. Jüttner, A. Khamseh, F. Sanfilippo, and J. T. Tsang, J. High Energy Phys. 12 (2017) 008 .

[55] A. Bazavov et al., Phys. Rev. D 98, 074512 (2018).

[56] P. A. Boyle, L. Del Debbio, N. Garron, A. Juttner, A. Soni, J. T. Tsang, and O. Witzel (RBC/UKQCD Collaboration), arXiv:1812.08791.

[57] F.-Z. Peng, M.-J. Yan, M. Sánchez Sánchez, and M. P. Valderrama, arXiv:2011.01915.

[58] M. Wang, arXiv:2012.10380.

[59] H.-X. Chen, W. Chen, X. Liu, and X.-H. Liu, arXiv:2011 .01079 .

[60] Z.-G. Wang, arXiv:2011.05102. 